\title{
Human Alpha Rhythms During Visual Delayed Choice Reaction Time Tasks: A Magnetoencephalography Study
}

\author{
Claudio Babiloni, ${ }^{-3 *}$ Fabio Babiloni, ${ }^{1,4}$ Filippo Carducci, ${ }^{1-3}$ \\ Febo Cincotti, ,,4 Claudio Del Percio,' Stefania Della Penna, 5,6 \\ Raffaella Franciotti, ${ }^{5,6}$ Sandro Pignotti,' Vittorio Pizzella, 5,6 \\ Paolo Maria Rossini, ${ }^{2,3,7}$ Elisabetta Sabatini, ${ }^{3,8}$ Kathya Torquati, ${ }^{5,6}$ \\ and Gian Luca Romani ${ }^{5,6}$ \\ ${ }^{1}$ Dipartimento di Fisiologia Umana e Farmacologia, Università La Sapienza, Rome, Italy \\ ${ }^{2}$ IRCCS S Giovanni di Dio, Via Pilastroni, Brescia, Italy \\ ${ }^{3}$ AFaR.-Dip. di Neuroscienze, S. Giovanni Calibita, Fatebenefratelli Isola Tiberina, Rome, Italy \\ ${ }^{4}$ IRCCS Fondazione Santa Lucia, Rome, Italy \\ ${ }^{5}$ Dipartimento di Scienze Cliniche e Bioimmagini, Università G. D'Annunzio, Chieti, Italy \\ ${ }^{6}$ ITAB-Fondazione "Università G. D' Annunzio," Chieti, Italy \\ ${ }^{7}$ Clinica Neurologica, Campus Biomedico, Università di Roma, Rome, Italy \\ ${ }^{8}$ Unità di Neuropsicologia, Policlinico Gemelli, Università Cattolica di Roma, Rome, Italy
}

\begin{abstract}
Magnetoencephalography (MEG) includes fast and comfortable recording procedures very suitable for the neurophysiological study of cognitive functions in aged people. In this exploratory MEG study in normal young adults, we tested whether very simple short-term memory (STM) demands induce visible changes in amplitude and latency of surface $\alpha$ rhythms. Two delayed response tasks were used. In the STM condition, a simple cue stimulus (one bit) was memorized along a brief delay period (3.5-5.5 s). In the control (no short-term memory; NSTM) condition, the cue stimulus remained available along the delay period. To make extremely simple the tasks, the explicit demand was visuospatial but the retention could be also based on phonological and somatomotor coding. Compared to the control condition, the amplitude of the $\alpha 1$ (6-8 $\mathrm{Hz})$ ERD decreased in the left hemisphere, whereas the amplitude of the $\alpha 2(8-10 \mathrm{~Hz})$ and $\alpha 3(10-12 \mathrm{~Hz})$ event-related desynchronization (ERD) increased in right and left parietal areas, respectively. Furthermore, the latency of the $\alpha$ ERD peak was slightly but significantly $(P<0.05)$ later in STM compared to control condition. In conclusion, whole-head MEG technology and very simple STM demands revealed significant changes of human neuromagnetic $\alpha$ rhythms in normal young adults. Hum Brain Mapp 24:184-192, 2005.
\end{abstract}

๑ 2004 Wiley-Liss, Inc.

Key words: short-term memory; delayed response tasks; cerebral cortex; magnetoencephalography; $\alpha$ rhythm; event-related desynchronization

Contract grant sponsor: Telethon Onlus Foundation; Contract grant number: E.C0985.

*Correspondence to: Dr. Claudio Babiloni, Dipartimento di Fisiologia Umana e Farmacologia, Università degli Studi di Roma "La Sapienza," P.le Aldo Moro 5, 00185 Rome, Italy.

E-mail: claudio.babiloni@uniromal.it

Received for publication 9 March 2004; Accepted 28 June 2004

DOI: 10.1002/hbm.20079

Published online in Wiley InterScience (www.interscience.wiley. com).

\section{INTRODUCTION}

Short-term memory (STM) can be investigated easily by the so-called "delayed choice" reaction time task [GoldmanRakic, 1987, 1988, 1991, 1995]. Subject has to retain a "cue" stimulus and a related motor response across a delay period up to an imperative "go" stimulus. In the STM variant of the task, the cue rapidly disappears. In the no short-term memory (NSTM) variant, the cue is maintained along the whole delay period. With respect to "match-to-sample" and "nback" working memory paradigms, the delayed choice reaction time task includes no online probe stimulus to be matched with the memorized cue. 


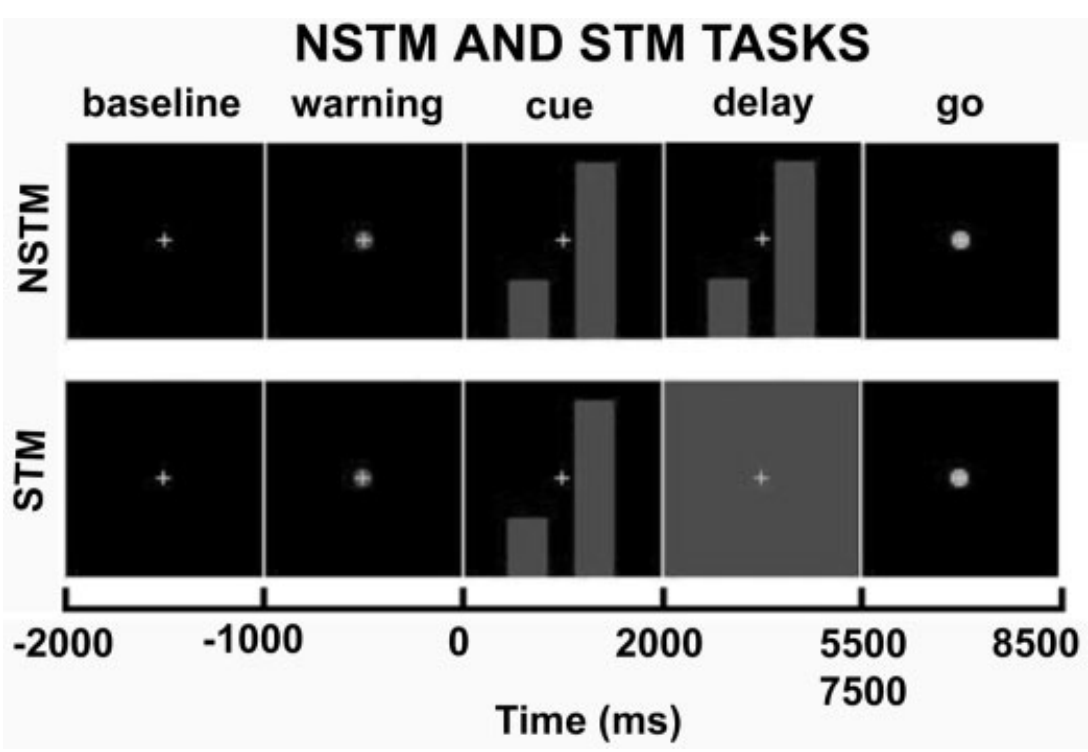

Figure I.

Set-up and experimental tasks. The experiment included a shortterm memory (STM) task compared with a control no short-term memory (NSTM) task. The sequence of events for the STM condition was as follows: (I) a cross at the center of the monitor as baseline lasting I s; (2) a cross surrounded by a red circle at the center of the monitor as a visual warning stimulus lasting I s; (3) a couple of vertical bars as a visual cue stimulus lasting $2 \mathrm{~s}$; (4) a blank screen as a delay period lasting 3.5-5.5 s; (5) a green circle

Cortical electroencephalographic (EEG) rhythms during delayed response tasks have been explored recently in young subjects [Babiloni et al., 2004a; Bastiaansen et al., 2002; Filipovic et al., 2001]. There were significant changes of $\alpha(\sim 6-12 \mathrm{~Hz})$ rhythms during the delay period, as a reflection of the desynchronizing drive of the thalamus on cortex [Filipovic et al., 2001; Pfurtscheller and Lopes da Silva, 1999].

The delayed choice reaction time tasks are simpler than "delayed match-to-sample" and "n-back" working-memory (WM) paradigms; therefore, they might be used in the neurophysiological study of physiological or pathological aging [Goldman-Rakic, 1991; Harrington et al., 2000; Roux et al., 1994; Servan-Schreiber et al., 1996]. In this regard, a recent high-resolution EEG study (using simple delayed response tasks) has shown that the amplitude of frontoparietal $\alpha$ event related desynchronization (ERD) were stronger in normal young than elderly subjects [Babiloni et al., 2004b]. The only obstacle for the application to pathological aging of that protocol is the long procedure for the montage of $48-128$ EEG electrodes.

Magnetoencephalography (MEG) includes fast and comfortable recording procedures very suitable for the neurophysiological study of cognitive functions in aged people. In this exploratory study, we tested whether a very simple short-term memory (STM) demand induces visible changes in amplitude and latency of neuromagnetic $\alpha$ rhythms in young adults. at the center of the monitor as a go stimulus lasting I s; and (6) a finger movement as a motor response. Subjects had to click the left mouse button if the taller bar was at the left monitor side, or the right mouse button if the taller bar was at the right monitor side. Compared with the STM task, the NSTM task had visual cue stimuli lasting up to the go stimulus. The zero time was the onset of the cue stimulus.

\section{SUBJECTS AND METHODS}

\section{Subjects}

The experiments were carried out in 20 healthy, righthanded (Edinburgh Inventory) young adult volunteers (mean age $=25$ years; range $=19-42$ years). They gave their informed written consent according to the declaration of Helsinki. The local institutional ethics committee approved general procedures.

\section{Experimental Tasks}

Subjects were seated in a comfortable reclining armchair placed in a dimly lit, sound-damped, and electrically shielded room. They kept their forearms resting on armchairs, with right index finger resting between two buttons spaced $6 \mathrm{~cm}$ apart. A computer monitor was placed in front of the subjects $(\sim 100 \mathrm{~cm})$. The STM task (Fig. 1) comprised a sequence of baseline stimulus ( 0.7 degrees cross at the center of the monitor for $1 \mathrm{~s}$ ), visual warning stimulus (the cross was surrounded by a red circle for $1 \mathrm{~s}$ ), visual cue stimulus (two vertical bars large $\sim 2$ degrees and height 2.5-7 degrees for $2 \mathrm{~s}$ ), delay period (blank screen for 3.5-5.5 s), go stimulus (a green circle appeared for $1 \mathrm{~s}$ ), and right finger movement to press the proper button of a custommade device. This device had two large buttons approximately $10 \mathrm{~cm}$ distant from one another, which were elec- 
tronically connected to the mouse of the computer giving the visual stimuli. Subjects had to click the left button if the taller bar (cue stimulus) was at the left monitor side, whereas they had to click the right button if the taller bar was at the right monitor side. In the no STM (NSTM) condition, the visual cue stimulus was delivered up to the go stimulus. The zero time was the onset of the cue stimulus. Two trial blocks for each condition were pseudorandomly intermingled (block $=50$ single trials; 2 min pause). Subjects were told in advance if the block was NSTM or STM. The global luminance of the monitor was kept practically uniform across the two conditions (i.e., the changes of the scene were limited to the form) and was measured directly on the monitor by a Tektronic J17 and J1800 Series LumaColor Photometer. In the perspective of future dementia studies, subjects were free to use any memorization strategy including visuospatial imagery, somatomotor preparation, and mental "phonological" coding and rehearsal. Before the recording session, a training of approximately $10 \mathrm{~min}$ was performed to familiarize the subjects with the experimental apparatus and tasks.

\section{MEG Recordings}

MEG data were recorded in continuous mode $(0.1-60 \mathrm{~Hz}$ bandpass, $250 \mathrm{~Hz}$ digital sampling) using the whole head system operating at the University of Chieti (ITAB). This system was equipped with 165 integrated superconducting quantum interface devices (SQUID) magnetometers (sensors noise of about $5 \mathrm{fT} / \sqrt{\mathrm{Hz}}, 153$ of which were placed over a helmet-shaped support providing whole scalp coverage. The remaining 12 SQUID magnetometers were used as reference channels for the software subtraction of the magnetic field noise. The position of the head with respect to the sensors, before and after each stimulation session, was determined by placing four coils on the subject head. The location of the coils and the anatomical landmarks on the subject head were digitized by means of a 3-D digitizer (Polhemus; 3Space Fastrak, VT, USA).

Surface electromyography activity was bilaterally recorded from extensor digitorum muscles (1-60 Hz bandpass, $250 \mathrm{~Hz}$ digital sampling) via disk electrodes in a bellytendon montage, to monitor operant movement as well as mirror movements and involuntary muscle activations.

\section{Off-line Preliminary Data Analysis}

The collected MEG data were segmented in single trials each spanning from $-2.5 \mathrm{~s}$ to $+11.5 \mathrm{~s}$, the zero time being the onset of the cue stimulus (i.e., vertical bars). Two independent experimenters executed off-line preliminary data analysis (inter-rater reliability higher than 95\%). The MEG single trials were discarded when associated with: (1) wrong cognitive performance; (2) mirror or involuntary finger movements of the non-operant side (about 14\%); and (3) inadvertent motor acts and slight muscle activation of the operant side before the go stimulus. The rejection of the single trials contaminated by blinking and eye movements was based on the analysis of the frontal MEG data. In 2 of 20 subjects, the number of artifact-free MEG single trials was

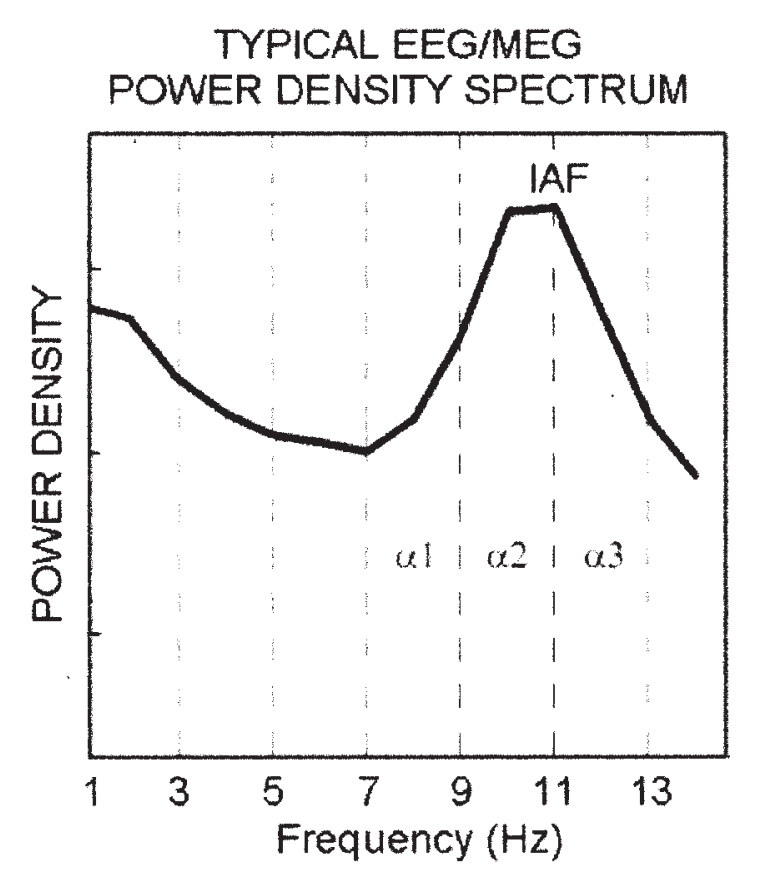

Figure 2.

Model of a typical frequency spectrum computed from MEG (EEG) data. Frequency bands of interest were $\alpha \mathrm{I}, \alpha 2$, and $\alpha 3$, which were computed according to a well-known procedure based on the detection of individual $\alpha$ frequency (IAF) peak (see Subjects and Methods for further explanations).

lower than $30 \%$ and their data were not employed for the final data analysis. Therefore, 18 subjects surpassed the preliminary MEG data analysis and were used for the further data analyses.

After this off-line preliminary data analysis, the individual artifact-free MEG single trials were 62 ( \pm 5 standard error [SE]) for the STM task and 61 ( \pm 5 SE) for the NSTM task, adequate for the study of ERD/ERS processes in cognitive tasks according to previous studies [Babiloni et al., 2004b,c; Okada and Salenius, 1998]. No inter-condition statistical difference was found by repeated measures ANOVA analysis $(F[1,17]=0.089 ; P=0.755)$.

Motor reaction time after the go stimulus was computed for all artifact-free EEG single trials of the NSTM versus STM conditions. It served as an index of the sustained attention across the trial in the two conditions.

\section{Determination of Individual $\alpha$ Bands}

The power spectrum analysis was based on a standard FFT approach using Welch technique and Hanning windowing function. Figure 2 shows a typical frequency spectrum computed from MEG (or EEG) data. For the determination of the individual $\alpha$ bands, an anchor frequency was selected according to literature guidelines [Klimesch, 1996, 1999; Klimesch et al., 1998]. This is the frequency of the so-called individual $\alpha$ frequency (IAF) peak, defined as the 


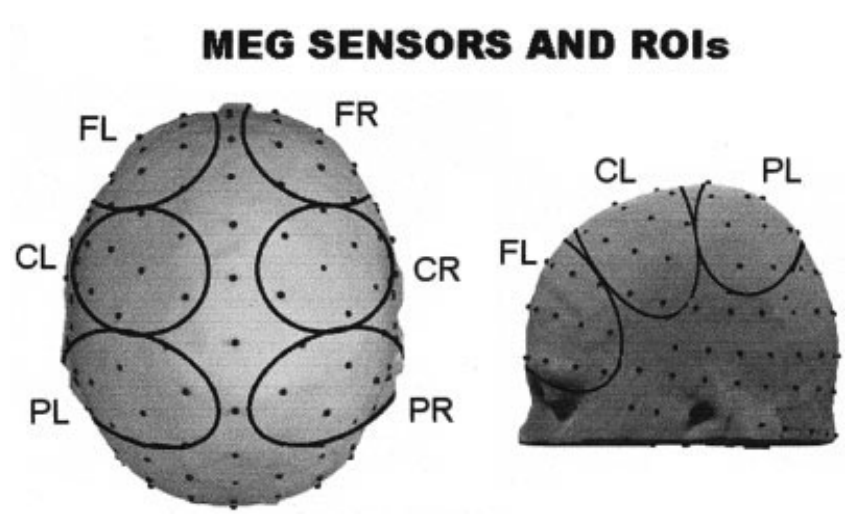

Figure 3.

The position of MEG sensors over a scalp model constructed by averaging the magnetic resonance images (I52 subjects) recorded at the Brain Imaging Center of Montreal Neurological Institute (SPM96). For illustrative purposes, the regions of interest (FL, left frontal; FR, right frontal; CL, left central; $C R$, right central; PL, left parietal; PR, right parietal) are also represented over this model.

frequency showing the higher power density at the 6-12 Hz range. With reference to the IAF, the frequency bands of interest were as follows: $\alpha 1$ as IAF- $4 \mathrm{~Hz}-\mathrm{IAF}-2 \mathrm{~Hz}, \alpha 2$ as IAF- $2 \mathrm{~Hz}-\mathrm{IAF}$, and $\alpha 3$ as IAF-IAF $+2 \mathrm{~Hz}$. As a result, the mean $( \pm S E)$ peak of the IAF was $10.05 \mathrm{~Hz}( \pm 0.25)$ for both NSTM and STM conditions.

\section{Computation of Event-Related Desynchronization}

To quantify the event-related percentage reduction of the $\alpha$ oscillations, a standard procedure for the computation of the event-related desynchronization (ERD) was used, [Pfurtscheller and Aranibar, 1979; Pfurtscheller and Neuper, 1994; Pfurtscheller et al., 1997]. The ERD was computed for the $\alpha 1, \alpha 2$ and $\alpha 3$ bands, based on the individual determination of the IAF. In brief, the MEG time series were bandpassed (Bartlett function), squared, averaged across 125 msec periods (to 8 samples/s), and averaged across all single trials. The ERD was defined as a percentage decrease of the instant power density at the "event" compared to a "pre-event" baseline (from -2 to $-1 \mathrm{~s}$ ). Remarkably, no correction for the visual evoked potentials (VEPs) was carried out, so that band power calculation theoretically included both non-phase-locked and phase-locked MEG components.

The individual ERD data sets were then interpolated by a spline function [Babiloni et al., 1995], to obtain ERD data sets at theoretical 153 sensor sites disposed over a 3-D "quasirealistic" scalp model (i.e., approximating each "realistic" individual head model). This template model was constructed based on the magnetic resonance data of 152 subjects, digitized at the Brain Imaging Center of the Montreal Neurological Institute (SPM96). Figure 3 shows for illustrative purposes the "quasi-realistic" scalp model, the position of the 153 sensor sites, and the inclusion of these sensors in 6 macro-regions of interest (ROI) such as left frontal (FL), right frontal (FR), left central (CL), right central (CR), left parietal (PL), and right parietal (PR).

\section{Measurement of ERD Latency and Amplitude}

The $\alpha$ ERD waveforms were analyzed to measure the ERD peak during the delay period. The ERD peak was defined as the absolute maximum ERD value during the delay period (it had to be also statistically significant at $P<0.05$ sign test). The ERD peak was independently recognized by two independent experiments (inter-rater reliability higher than 95\%) within the frontal, central, and parietal ROIs. The ERD/ERS amplitude was automatically measured for each experimental condition at each of the 153 sensors. The ROI having the maximal ERD value was considered as a reference for the latency of the topographical mapping.

\section{Statistical Analysis for NSTM and STM Comparisons}

The statistical analysis of the data was carried out by analysis of variance (ANOVA) for repeated measures. Mauchly's test evaluated the sphericity assumption and correction of the degrees of freedom was made by GreenhouseGeisser procedure when necessary. Duncan test was used for the post-hoc comparisons $(P<0.05)$.

For the evaluation of the correct responses, the ANOVA used the factor Condition (NSTM, STM). For the evaluation of the behavioral errors, the ANOVA used the factors Condition (NSTM, STM) and Error type (Wrong responses, Anticipated response respect to the go stimulus, Delayed response exceeding $2.5 \mathrm{~s}$ after the go stimulus). In 4 of 18 subjects instrumental problems prevented the estimation of the percentages of the specific kind of behavioral errors. Consequently, only 14 subjects were used for the statistical analysis of the behavioral data.

For the evaluation of the movement reaction time after the go stimulus, the ANOVA used the factor Condition (NSTM, STM).

For the evaluation of the $\alpha$ ERD latency, the ANOVA used the factors Condition (NSTM, STM) and Band ( $\alpha 1, \alpha 2, \alpha 3)$.

For the evaluation of the ERD amplitude, the ERD values were averaged across the electrodes belonging to the same ROI. The mean ERD value served as an input to three repeated ANOVA measures; one ANOVA for each band of interest (i.e., $\alpha 1, \alpha 2$ and $\alpha 3$ ). The ANOVA factors were Condition (STM, STM) and ROI (FL, FR, CL, CR, PL, PR).

\section{RESULTS}

\section{Behavioral Results}

NSTM and STM tasks were equally simple as revealed by the correct responses; $97.8 \%$ ( $\pm 2 \mathrm{SE}$ ) for the NSTM condition and $97.7 \%$ ( $\pm 2 \mathrm{SE}$ ) for the STM condition. Nevertheless, ANOVA pointed to a main effect for the factor Error type $(F[2,26]=22.15 ; P=0.0001)$ indicating that the anticipated responses (1.75\% for NSTM and $1.2 \%$ for STM) were more 

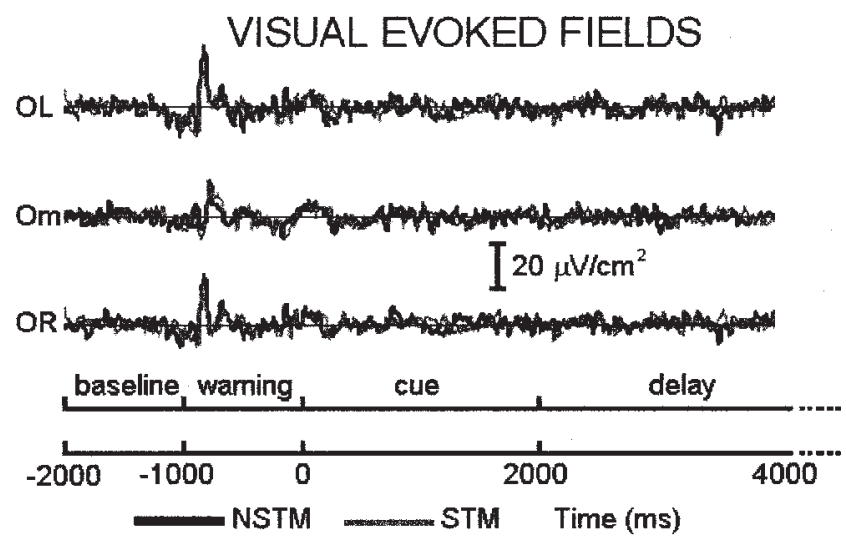

Figure 4.

The grand average waveforms of visual evoked fields $(0.5-30 \mathrm{~Hz}$ bandpass) for the NSTM and the STM conditions. These waveforms refer to representative occipital sensors sites.

frequent than the wrong $(P=0.0003)$ and delayed $(P$ $=0.0006$ ) responses.

The reaction time (as an. index of the tonic attention) was slightly shorter during the NSTM ( $574 \mathrm{~ms} \pm 23 \mathrm{SE}$ ) than STM (591 $\mathrm{ms} \pm 22 \mathrm{SE}$ ) condition, but this difference did not reach statistical significance $(F[1,17]=0.8315 ; P=0.375)$.

\section{Temporal Evolution of Visual Evoked fields}

Figure 4 shows the group waveforms of the visual evoked fields (0.5-30 Hz bandpass) during the visual warning stim- ulus, cue stimulus, and delay period (i.e., only the first $2 \mathrm{~s}$ of this period) of the NSTM and STM tasks. Representative occipital sensors were considered. Notably, the amplitude of the visual evoked fields returned at baseline values before the end of the cue period (note that the offset of the cue stimulus induced no visual evoked fields). Therefore, these evoked fields could not substantially affect the ERD/ERS during the delay period.

\section{Temporal Evolution of $\alpha$ ERD}

Figure 5 shows the group ERD waveforms of the $\alpha 1, \alpha 2$, and $\alpha 3$ for the NSTM and STM conditions. These waveforms refer to the trials with correct responses and are representative of the frontal and parietal sensors. The analysis of temporal evolution of the $\alpha$ ERD was especially focused on the period of interest, i.e., the delay period. The $\alpha$ ERD waveforms peaked within the first $2 \mathrm{~s}$ of the delay period (i.e., in Fig. 5 the range of $2 \mathrm{~s}$ is represented by two red vertical lines) and had maximal amplitude at parietal sensors. The $\alpha 1, \alpha 2$, and $\alpha 3$ ERD peaks were sustained and statistically significant in all 18 subjects.

Table 1 reports the mean ( \pm SE) latency of $\alpha$ ERD peaks at parietal sensors (i.e., zero time is the onset of the cue stimulus). The $\alpha$ ERD peaks ranged from 2,377-2,577 $\mathrm{ms}$ for the NSTM condition and from 2,566-2,755 ms for the STM condition. ANOVA for the $\alpha$ ERD latency showed a main effect for the factor Condition $(F[1,17]=4.55 ; P=0.045)$ indicating that the $\alpha$ ERD peak was later for the STM than NSTM condition.
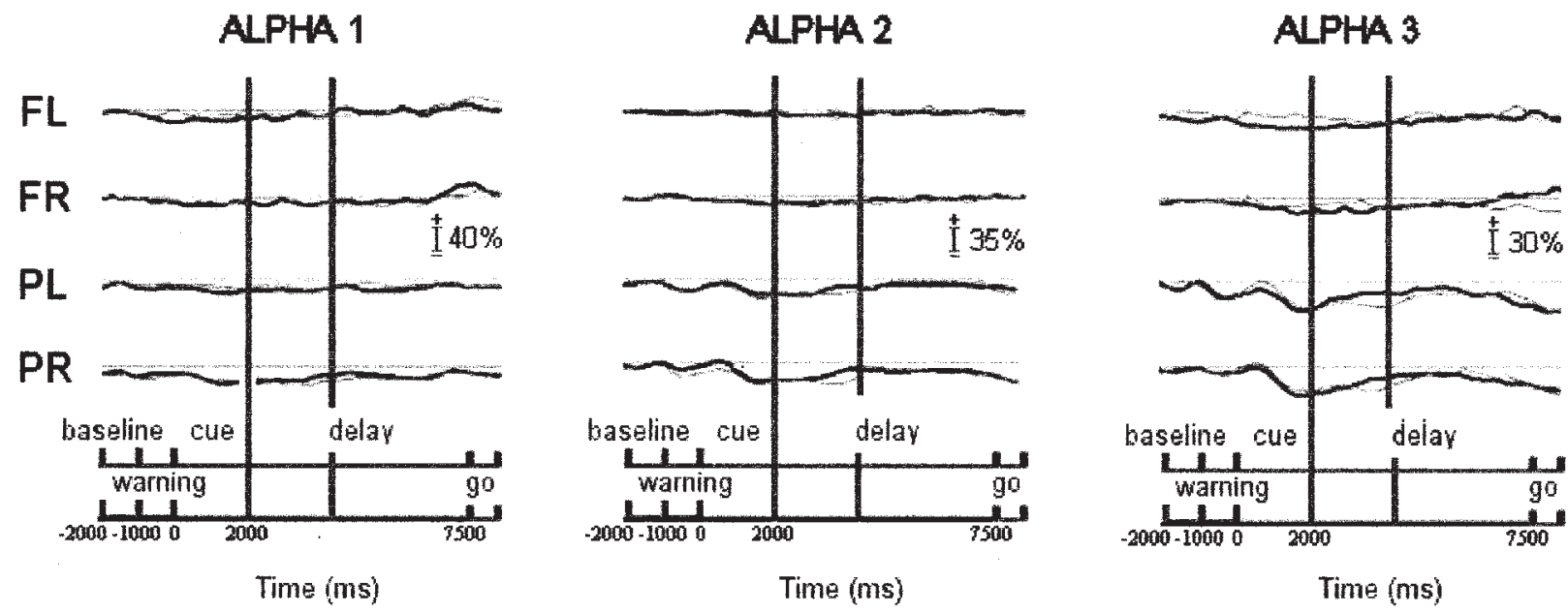

NSTM STM

Figure 5.

The grand average waveforms of the $\alpha \mathrm{I}, \alpha 2$, and $\alpha 3$ ERD in the NSTM and the STM conditions. These waveforms refer to representative frontal and parietal sensor sites. The red vertical lines indicate the first 2 of the delay period where the $\alpha$ ERD peaked. 
TABLE I. Latency of $\alpha \mathrm{I}, \alpha 2$, and $\alpha 3$ ERD peaks for no short-term memory and short-term memory conditions

\begin{tabular}{lcc}
\hline & \multicolumn{2}{c}{ Latency $(\mathrm{ms})$} \\
\cline { 2 - 3 } ERD peak & No short-term memory & Short-term memory \\
\hline$\alpha 1$ & $2,422 \pm 104$ & $2,755 \pm 134$ \\
$\alpha 2$ & $2,377 \pm 64$ & $2,566 \pm 120$ \\
$\alpha 3$ & $2,577 \pm 121$ & $2,566 \pm 109$ \\
\hline
\end{tabular}

Values are given as means \pm SE. Zero time indicates the onset of the visual cue stimulus (two vertical bars).

ERD, event-related desynchronization.

\section{Spatial Distribution of ERD Peaks}

Figure 6 shows the topographical maps of the group $\alpha 1$, $\alpha 2, \alpha 3$ ERD peaks plotted in Figure 5 for illustrative purpose only. For the NSTM condition, the maximum $\alpha$ ERD (all bands) was observed in the parietal and central areas. For the STM condition, the ERD was recognized in right centroparietal area at $\alpha 2$ and in left parietal area at $\alpha$ 3 . Of note, the present topographic maps did not represent the cortical sources of the $\alpha$ ERD.

\section{Statistical Analysis of ERD Peak}

All three separate ANOVA for $\alpha 1,2$, and 3 ERD amplitude (18 subjects) showed a statistical interaction $(F[5,85]=$ 3.24; $P=0.01$ ) between the factors Condition (NSTM, STM) and ROI (FL, FR, CL, CR, PL, PR). Figure 7 illustrates the

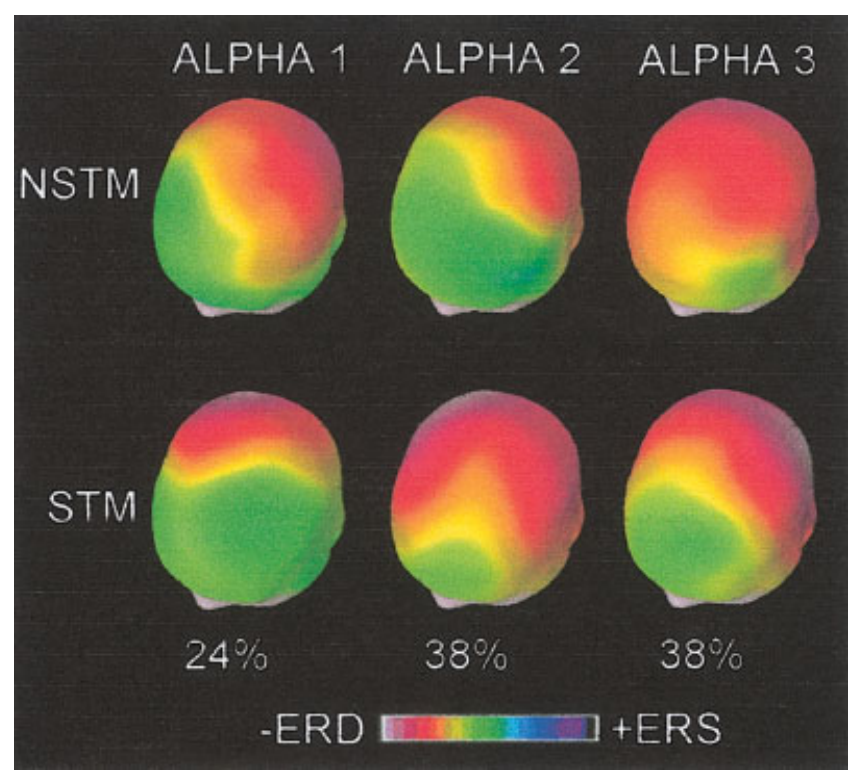

Figure 6.

Three-dimensional color maps of $\alpha \mathrm{I}, \alpha 2$, and $\alpha 3$ ERD peaks for the NSTM and STM tasks. Color scale: maximum ERD and eventrelated synchronization (ERS) are coded in white and violet, respectively. The maximal (\%) value of the ERD/ERS is reported.

\section{ANOVA INTERACTION OF TASK AND ROI}

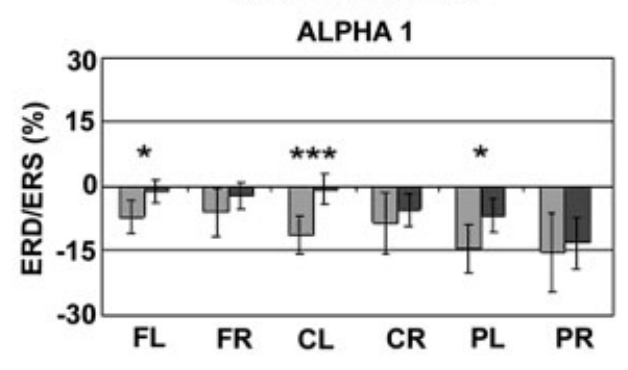

ALPHA 2
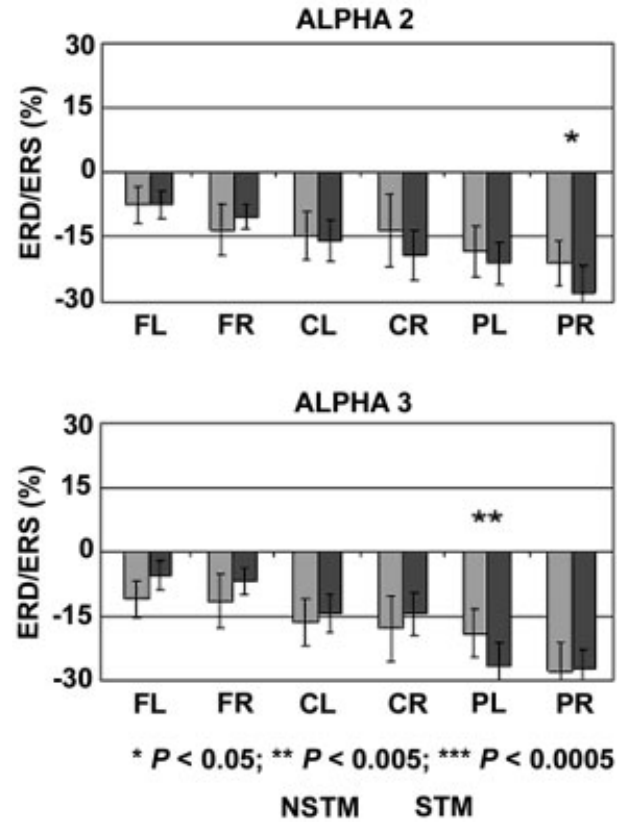

Figure 7.

Across-subject means ( $\pm \mathrm{SE}$ ) of the ERD amplitudes as provided by ANOVA design. In particular, these means refer to a statistical interaction between the factors Condition (NSTM, STM), and ROI (FL, FR, CL, CR, PL, PR). The $\alpha$ I (top), $\alpha 2$ (middle), and $\alpha 3$ (bottom) values were used as dependent variables The results of the Duncan post-hoc testing are indicated by $* P<0.05$, ** $P$ $<0.005$, or $* * * P<0.0005$.

mean $( \pm \mathrm{SE})$ of the $\alpha 1$ ERD representing these statistical results. For $\alpha 1$, Duncan post-hoc testing indicated that the ERD peak was stronger for the NSTM than for the STM condition in left frontal $(P=0.043)$, left central $(P=0.0005)$, and left parietal $(P=0.048)$ areas. For $\alpha 2$, the ERD peak was stronger for the STM than for the NSTM in right parietal area $(P=0.048)$. For $\alpha 3$, the ERD peak was stronger for the STM than for the NSTM in left parietal area $(P=0.005)$.

Control ANOVA analyses were designed to exclude that the above findings were due to a different baseline band power of $\alpha$ oscillations. The design included the factors Condition (NSTM, STM) and ROI (FL, FR, CL, CR, PL, PR). There was no statistical effect $(P>0.25)$, confirming the validity of the ERD results. 


\section{DISCUSSION}

\section{Methodological Remarks}

In the present exploratory MEG study, surface $\alpha$ ERD was investigated. The procedure required no computational assumption such as the number of equivalent dipoles or regularization parameters [Babiloni et al., 2001a] and was successfully applied in prior investigations [Babiloni et al., 1999, 2000, 2001b, 2002; Gevins and Smith, 2000; Gevins et al., 1998; McEvoy et al., 2001; Okada and Salenius, 1998]. As a limitation, $\alpha$ ERD sources could be localized. For example, a single cortical source generates extracranial dipolar neuromagnetic fields whose surface maxima do not exactly overlay the location of that source, namely the maximal influx is located at one side of the source and the maximal outflux is located at the other side. Furthermore, the activity of near cortical areas may be not disentangled. For these reasons, the macroregions of interest of the present study just referred to the localization of the sensors at the surface scalp level and the detected $\alpha$ ERD could be generated not only by the underlying cortical areas but also by the contiguous cortical areas. The present findings tested whether a very simple STM demand induces visible changes in amplitude and latency of surface $\alpha$ ERD in young adults. This may be of interest for possible applications on aging in clinical environments with poor technological resources for MEG source analysis. Of note, the spatial resolution of MEG data can be enhanced by several mathematical techniques including those illustrated by Hari group [Hari and Forss, 1999; Hari et al., 1988, 1997; Portin et al., 1999; Salenius et al., 1995; Stenbacka et al., 2002] as well as Bastiaansen and Knosche [2000].

The present $\alpha$ ERD could not dissociate the two main processes occurring during the delay period, i.e., the retention and the motor preparation [Rowe et al., 2000]. However, the motor preparation may not confound the results for at least three reasons. First, the motor preparation was paired for the NSTM and STM conditions, in which the time intervals, go stimulus, and motor demands were identical. Second, the delay period between the cue and go stimuli varied trial-by-trial (3.5-5.5 s), to discourage a pre-stimulus motor preparation. Third, the $\alpha$ ERD did not progressively increase during the delay period as expected for a pure motor process. Indeed, the typical trend of the motor preparation is a progressive increase of the $\alpha$ ERD up to the movement execution [Hari et al., 1997; Pfurtscheller and Lopes da Silva, 1999].

The determination of the individual $\alpha$ bands followed the influential guidelines by Klimesch et al. [Klimesch, 1996, 1999; Klimesch et al., 1998]. That approach has provided a body of evidence that the analysis of individual EEG frequency bands can disclose invaluable information on brain rhythmicity [Klimesch, 1999; Kopruner et al., 1984; Niedermayer, 1993].

\section{Alpha ERD is Modulated by a Very Simple STM Load}

Compared to the control condition (delay period), the STM condition induced a weaker $\alpha 1$ ERD $(6-8 \mathrm{~Hz})$ in the left hemisphere as well as a prominent $\alpha 2(8-10 \mathrm{~Hz})$ and $\alpha$
$3(10-12 \mathrm{~Hz})$ ERD in the right and left parietal areas, respectively. Furthermore, the latency of the $\alpha$ ERD peaks was slightly but significantly $(P<0.05)$ later in STM compared to control (NSTM) condition, in line with a slowing of cognitive cortical processes due to the present simple STM demand.

In general, the result of a prominent $\alpha$ ERD in STM than NSTM condition confirms our previous EEG study using the same task on normal young subjects [Babiloni et al., 2004a]. According to the current view on $\alpha$ sub-bands [Klimesch, 1999], the wide $\alpha 1$ ERD might indicate a marked global visual attention during the control condition associated with the prolonged permanence of the stimulus crucial for the performance (i.e., cue visual stimulus). In the memory condition (STM), the offset of the cue stimulus induced no visible visual evoked magnetic fields. Therefore, it can be speculated that the disappearance of the cue stimulus into a red square did not synchronize the visual neural populations towards the generation of phase-locked visual evoked fields. The visual processing of the red square would operate at lower spatial and synchronization scales than those visible by MEG techniques.

In parallel, the $\alpha 2$ and 3 ERD in the parietal areas might be related to the retention processes characterizing the STM condition. However, the memory load was so slight (only one bit towards applications to aging) that no significant differences in the behavioral performance emerged. In precedence, previous neuroimaging studies have shown that the coding and rehearsal of visuospatial representations mainly impinge upon right posterior parietal cortex [Smith and Jonides, 1997], whereas left frontal and parietal areas could support a possible complementary verbalization in encoding, storage, and rehearsal processes [Fiez and Petersen, 1998].

The present results extend those of a recent low-resolution EEG study on delayed reaction time tasks in which low but not high $\alpha$ ERD has been reported [Bastiaansen et al., 2002]. In the present study, the warning (1 s) and cue (2 s) stimuli were relatively long, leading to no visual evoked response during the delay period. Consequently, this unmasked parallel $\alpha$ ERD occurring during the delay period. The present results complement previous high-resolution EEG evidence during n-back tasks showing a ERD [Gevins et al., 1997], which was stronger with heavy memory load [Gevins et al., 1997; Gevins and Smith, 2000; McEvoy et al., 2001]. The present evidence of high $\alpha$ ERD during the delay period is also in agreement with the findings of whole-head MEG recordings using a more demanding delayed response task [Okada and Salenius, 1998].

\section{CONCLUSIONS}

This exploratory MEG study in young adults showed an increment of the $\alpha$ ERD amplitude in a simple delayed response task with a slight memory demand (only one bit) with respect to the control condition. Furthermore, the latency of the $\alpha$ ERD peak was slightly but significantly ( $P$ $<0.05)$ later in STM than in the control condition These 
results confirm the working hypothesis that a whole-head MEG technology, which requires a quick procedure of sensors montage with respect to high-resolution electroencephalography, and a simple STM demand revealed changes in human neuromagnetic $\alpha$ rhythms. In future investigations, the use of delayed response tasks and MEG technique may be tested for the study of physiological and pathological aging, at least at the global level of populations. Indeed, these tasks may maximize the correct responses, to assure an acceptable signal-to-noise ratio of the neurophysiological signals to be compared in the young versus elderly subjects. Furthermore, the MEG recordings may reduce the duration of experimental session, to avoid fatigue/distraction in aged patients.

\section{ACKNOWLEDGMENTS}

We thank Prof. Fabrizio Eusebi, Chairman of the Biophysics Group of Interest of Rome I University, for his continuous support.

\section{REFERENCES}

Babiloni C, Babiloni F, Carducci F, Cappa S, Cincotti F, Del Percio C, Miniussi C, Morett DV, Rossi S, Sosta K, Rossini PM (2004a): Human cortical responses during one-bit short-term memory. A high-resolution EEG study on delayed choice reaction time tasks. Clin Neurophysiol 115:161-170.

Babiloni C, Babiloni F, Carducci F, Cappa S, Cincotti F, Del Percio C, Miniussi C, Moretti DV, Rossi S, Sosta K, Rossini PM (2004b): Human cortical rhythms during visual delayed choice reaction time tasks: a high-resolution EEG study on normal aging. Behav Brain Res 153:261-271.

Babiloni C, Babiloni F, Carducci F, Cappa S, Cincotti F, Del Percio C, Miniassi C, Moretti DV, Pasqualetti P, Rossi S, Sosta K, Rossini PM (2004c): Human cortical EEG rhythms during long-term episodic memory task. A high resolution EEG study of the HERA model. Neuroimage 21:1576-1584.

Babiloni C, Babiloni F, Carducci F, Cincotti F, Del Percio C, De Pino G, Maestrini S, Priori A, Tisei P, Zanetti O, Rossini PM (2000): Movement-related electroencephalographic reactivity in Alzheimer disease. Neuroimage 12:139-146.

Babiloni C, Babiloni F, Carducci F, Cincotti F, Rosciarelli F, Rossini P, Arendt-Nielsen L, Chen A (2001b): Mapping of early and late human somatosensory evoked brain potentials to phasic galvanic painful stimulation. Hum Brain Mapp 12:168-179.

Babiloni C, Babiloni F, Carducci F, Cincotti F, Rosciarelli F, ArendtNielsen L, Chen AC, Rossini PM (2002): Human brain oscillatory activity phase-locked to painful electrical stimulations: a multichannel EEG study. Hum Brain Mapp 15:112-123.

Babiloni F, Babiloni C, Fattorini L, Carducci F, Onorati P, Urbano A (1995): Performances of surface Laplacian estimators: a study of simulated and real scalp potential distributions. Brain Topogr 8:35-45.

Babiloni C, Carducci F, Cincotti F, Rossini PM, Neuper C, Pfurtscheller G, Babiloni F (1999): Human movement-related potentials vs desynchronization of EEG $\alpha$ rhythm: a high-resolution EEG study. Neuroimage 10:658-665.

Babiloni F, Carducci F, Cincotti F, Del Gratta C, Pizzella V, Romani GL, Rossini PM, Tecchio F, Babiloni C (2001a): Linear inverse source estimate of combined EEG and MEG data related to voluntary movements. Hum Brain Mapp 14:197-209.
Bastiaansen MCM, Knosche TR (2000): Tangential derivative mapping of axial MEG applied to event-related desynchronization research. Clin Neurophysiol 111:1300-1305.

Bastiaansen MCM, Posthuma D, Groot P, de Geus E (2002): Eventrelated $\alpha$ and theta responses in a visuo-spatial working memory task. Clin Neurophysiol 113:1882-1893.

Fiez JA, Petersen SE (1998): Neuroimaging studies of word reading. Proc Natl Acad Sci USA 95:914-921.

Filipovic SR, Jahanshahi M, Rothwell JC (2001): Uncoupling of contingent negative variation and $\alpha$ band event-related desynchronization in a go/no-go task. Clin Neurophysiol 112:13071315.

Gevins A, Smith ME, McEvoy L, Yu D (1997): High-resolution EEG mapping of cortical activation related to working memory: effects of task difficulty, type of processing, and practice. Cereb Cortex 7:374-385.

Gevins A, Smith ME, Leong H, McEvoy L, Whitfield S, Du R, Rush GI (1998): Monitoring working memory load during computerbased tasks with EEG pattern recognition methods. Hum Factors 40:79-91.

Gevins A, Smith ME (2000): Neurophysiological measures of working memory and individual differences in cognitive ability and cognitive style. Cereb Cortex 10:829-839.

Goldman-Rakic PS (1987): Circuitry of primate prefrontal cortex and regulation of behavior by representational memory. In: Plum F, editor. Handbook of physiology; nervous system, vol. V. Higher functions of the brain, part 1. Bethesda, MD: American Physiological Society. p 373-417.

Goldman-Rakic PS (1988): Topography of cognition: parallel distributed networks in primate association cortex. Annu Rev Neurosci 11:137-156.

Goldman-Rakic PS (1991): Prefrontal cortical dysfunctions in schizophrenia: the relevance of working memory. In: Carroll BJ, Bassett JE, editors. Psychopatholology and the Brain. New York: Raven Press. p 1-23.

Goldman-Rakic PS (1995): Cellular basis of working memory. Neuron 14:477-485.

Hari R, Joutsiniemi SL, Sarvas J (1988): Spatial resolution of neuromagnetic records: theoretical calculations in a spherical model. Electroencephalogr Clin Neurophysiol 71:64-72.

Hari R, Salmelin R, Mäkelä JP, Salenius S, Helle M (1997): Magnetoencephalographic cortical rhythms. Int J Psychophysiol 26:5162.

Hari R, Forss N (1999): Magnetoencephalography in the study of human somatosensory cortical processing. Philos Trans R Soc Lond B Biol Sci 354:1145-1154.

Harrington F, Saxby BK, McKeith IG, Wesnes K, Ford GA (2000): Cognitive performance in hypertensive and normotensive older subjects. Hypertension 36:1079-1082.

Klimesch W (1996): Memory processes, brain oscillations and EEG synchronization. Int J Psychophysiol 24:61-100.

Klimesch W, Doppelmayr M, Russegger H, Pachinger T, Schwaiger J (1998): Induced $\alpha$ band power changes in the human EEG and attention. Neurosci Lett 244:73-76.

Klimesch W (1999): EEG $\alpha$ and theta oscillations reflect cognitive and memory performance: a review and analysis. Brain Res Brain Res Rev 29:169-195.

Kopruner V, Pfurtscheller G, Auer LM (1984): Quantitative EEG in normals and in patients with cerebral ischemia. Prog Brain Res 62:29-50

McEvoy LK, Pellouchoud E, Smith ME, Gevins A (2001): Neurophysiological signals of working memory in normal aging. Brain Res Cogn Brain Res 11:363-376. 
Niedermayer E (1993): The normal EEG in waking adult. In: Niedermayer E, Lopez da Silva, editors. Electroencephalography: basic principles, clinical application and related fields. Baltimore: Williams and Wilkins. 131-152.

Okada YC, Salenius S (1998): Roles of attention, memory and motor preparation in modulating human brain activity in a spatial working memory task. Cereb Cortex 8:80-96.

Pfurtscheller G, Aranibar A (1979): Evaluation of event-related desynchronization (ERD) preceding and following voluntary self-paced movement. Electroencephalogr, Clin Neurophysiol 46:138-146.

Pfurscheller G, Klimeschw (1992): Functional topography during a visuoverbal judgement task studied with event-related desynchronization mapping. J Clin Neurophysiol 9:120-131.

Pfurtscheller G, Neuper C (1994): Event-related synchronization of $\mathrm{mu}$ rhythm in the EEG over the cortical hand area in man. Neurosci Lett 174:93-96.

Pfurtscheller G, Neuper C, Flotzinger D, Pregenzer M (1997): EEG-based discrimination between imagination of right and left hand movement. Electroencephalogr Clin Neurophysiol 103:642-651.

Pfurtscheller G, Lopez da Silva F (1999): Event-related EEG/MEG synchronization and desynchronization: basic principles. Clin Neurophysiol 110:1842-1857.
Portin K, Vanni S, Virsu V, Hari R (1999): Stronger occipital cortical activation to lower than upper visual field stimuli. Neuromagnetic recordings. Exp Brain Res 124:287-294.

Roux S, Hubert I, Lenegre A, Milinkevitch D, Porsolt RD (1994): Effects of piracetam on indices of cognitive function in a delayed alternation task in young and aged rats. Pharmacol Biochem Behav 49:683-688.

Rowe JB, Toni I, Josephs O, Frackowiak RS, Passingham RE (2000): The prefrontal cortex: response selection or maintenance within working memory Science 288:1656-1660.

Salenius S, Kajola M, Thompson WL, Kosslyn S, Hari R (1995): Reactivity of magnetic parieto-occipital $\alpha$ rhythm during visual imagery. Electroencephalogr Clin Neurophysiol 95:453- 462.

Servan-Schreiber D, Cohen JD, Steingard S (1996): Schizophrenic deficits in the processing of context. A test of a theoretical model. Arch Gen Psychiatry 53:1105-1112.

Smith EE, Jonides J (1997): Working memory: a view from neuroimaging. Cognit Psychol 33:5-42.

Stenbacka L, Vanni S, Uutela K, Hari R (2002): Comparison of minimum current estimate and dipole modeling in the analysis of simulated activity in the human visual cortices. Neuroimage 16:936-943. 